\title{
Application of the modified stented elephant trunk procedure in type B aortic dissection
}

\author{
Honglei Zhao, Tao Bai, Jinrong Xue, Lizhong Sun, Yongmin Liu \\ Department of Cardiovascular Surgery, Beijing Anzhen Hospital, Capital Medical University, Beijing Institute of Heart, Lung and Blood Vessel \\ Diseases, Beijing Aortic Disease Center, Beijing Engineering Research Center for Vascular Prostheses, Beijing 100029, China \\ Contributions: (I) Conception and design: Y Liu, L Sun, H Zhao; (II) Administrative support: Y Liu, L Sun; (III) Provision of study materials or \\ patients: H Zhao, T Bai, J Xue, L Sun, Y Liu; (IV) Collection and assembly of data: H Zhao, T Bai, J Xue; (V) Data analysis and interpretation: H \\ Zhao, T Bai, J Xue; (VI) Manuscript writing: All authors; (VII) Final approval of manuscript: All authors. \\ Correspondence to: Yongmin Liu. Department of Cardiovascular Surgery, Beijing Anzhen Hospital, Capital Medical University, No. 2 Anzhen Road, \\ Beijing 100029, China. Email: liuyongmin100@sina.com.
}

Background: Considering the progressive nature of complicated Stanford type B aortic dissection (TBAD), operation must anticipate the need for later interventions. Recently we have used a modified stent elephant trunk (SET) procedure to treat these patients. And we review the indications, considerations for planning, and operative details for this this modified SET procedure.

Methods: Ten patients (seven males and three females) with complicated TBAD underwent a modified SET procedure in Anzhen Hospital, Beijing in the period between January 2019 and December 2019. The patients, whose ages ranged from 29-52 (42 \pm 7.23$)$, suffered complications with hypertension. Under deep hypothermic circulatory arrest, all the patients received SET implantation via an incision of the aortic arch. Before the patients were discharged, a computed tomography angiography (CTA) was performed to ensure the safety and accuracy of the procedure.

Results: The average surgery time was 4.0-5.5 (4.65 \pm 0.47$)$ hours, cardiopulmonary bypass (CPB) time was 115-163 (138.6 \pm 15.64$)$ minutes, aortic cross-clamping time was $32-59(42 \pm 7.72)$ minutes, and selective cerebral perfusion (SCP) time was 15-32 (21.7 \pm 5.56$)$ minutes. The SCP flow and nasopharyngeal temperature were 5-8 $(6.8 \pm 1.23) \mathrm{mL} / \mathrm{kg} / \mathrm{min}$ and $23.5-26.8(25.22 \pm 0.96){ }^{\circ} \mathrm{C}$, respectively. During SCP, the blood pressure of the left upper limb was 20-31 (25.5 3.81$) \mathrm{mmHg}$. Aorta cannulation position: vascular graft was anastomosed to the innominate artery (IA) and left subclavian artery (LSA) in seven cases, and with IA, LSA, and the right femoral artery in three cases. Ventilation time was 5-15 $(8.8 \pm 2.94)$ h, retention time in ICU was one day, and post-operative hospitalization time was $6-8(6.9 \pm 0.74)$ days. None of the patients died, or experienced endoleakage or neurological complications.

Conclusions: The application of a modified stented elephant trunk procedure in the treatment of TBAD is safe and efficient.

Keywords: Modified stented elephant trunk procedure (SET); Stanford type B aortic dissection (TBAD); computed tomography angiography (CTA)

Submitted Jan 17, 2020. Accepted for publication Feb 25, 2020.

doi: $10.21037 /$ atm.2020.03.109

View this article at: http://dx.doi.org/10.21037/atm.2020.03.109

\section{Introduction}

Aortic dissection, a serious condition where the inner layer of the aorta tears apart, has an abysmal natural prognosis. The onset of aortic dissection exposes the patient to extremely lethal outcomes. Aortic dissection is sub-divided into Stanford type A and Stanford type B aortic dissection (TBAD) based on the sites involved. Moreover, surgery is needed to effectively treat Stanford type A aortic dissection, 
while controversy continues to surround the treatment of TBAD. In particular, the non-invasive thoracic endovascular aortic repair (TEVAR) procedure has become the preferred treatment for TBAD as opposed to open surgery and has profound healing effects for most of the patients (1). For some younger patients who have complicated TBAD, surgical treatment is carried out in the form of a stented elephant trunk procedure (2-4), which employs the right axillary artery as the preferred arterial cannulation site. The left subclavian artery (LSA) is anastomosed to the left common carotid artery. In this paper, we implemented this technique on some subjects to explore its safety and efficiency in clinical applications, using a $10 \mathrm{~mm}$ artificial blood vessel to achieve long-term patency rate.

\section{Methods}

\section{Clinical data}

We enrolled 10 subjects ( 7 males and 3 females), aged 29-52 $(42 \pm 7.23)$ years old, with complicated TABD, to undergo a modified SET procedure at Anzhen Hospital, Beijing, in the period from January 2019 to December 2019. The patients ranged in weight from $60-110(86.2 \pm 17.42) \mathrm{kg}$, including 5 patients $\geq 90 \mathrm{~kg}$, and each of them had hypertension-related complications. A stent graft was implanted into all of the patients through an aortic arch incision under hypothermic cardiopulmonary bypass (CPB). Before the patients were discharged, they were followed up with an aortic computed tomography angiography (CTA) examination. In complicated TABD, the primary laceration is located less than $1.5 \mathrm{~cm}$ from the LSA, or at the lesser curvature of the aortic arch, with insufficient or no landing zone at the distal aortic arch (5).

\section{Surgical methods}

For each of the patients, the operation took place under deep hypothermic circulatory arrest and antegrade selective cerebral perfusion (SCP). The patients were positioned in a supine position, before general anesthesia, disinfection, and median thoracotomy to free brachiocephalic artery branches were subsequently carried out. For patients whose weight $\geq 90 \mathrm{~kg}$, the right femoral artery was kept free for standby.

After heparinization, a $10 \mathrm{~mm}$ artificial blood vessel (Maquet, Rastatt, Germany) was taken and anastomosed with the innominate artery (IA) in an end-to-side manner. It was sutured continuously with 5-0 prolene suture with subsequent exhaustion. Then, an $8 \#$ imported artery cannula was inserted through the artificial blood vessel. A vent catheter was reserved at the vent hole joint, and for overweight patients, femoral artery cannula was simultaneously performed. Extracorporeal circulation was established through venous cannulation via the right atrium, and the right superior pulmonary vein was placed and directed into the left ventricle for venting.

In the cooling process, the base of the LSA was detached and closed using the sutures. The artificial blood vessel was anastomosed to the distal end of the subclavian artery in an end-to-end manner via the 5-0 prolene continuous suture. The previously reserved vent catheter was then applied to perfuse the subclavian artery. After ventricular fibrillation, the ascending aorta was cross-clamped, and for myocardial protection, the base was perfused with cardioplegia.

Once the nasopharyngeal temperature had dropped to about $25^{\circ} \mathrm{C}$, SCP was performed via artificial blood vessels, and the femoral artery cannulation was clamped. The perfusion flow was specified to maintain the blood pressure of the left upper limb at 20-40 $\mathrm{mmHg}$. The IA and the left common carotid artery were cross-clamped, undergoing distal circulatory arrest, and, via the aortic arch incision, the stent (Cronus $^{\circledR}$, MicroPort Medical, Shanghai, China) was implanted in an open antegrade manner. After the stent was released, the stented blood vessel was located at the distal aortic arch wall in the full-thickness and continuous circumferential suture.

A series of reviving steps were carried out following the surgical procedure, including exhaustion, paying off oxygen debt, rewarming, and opening the ascending aorta. These were followed by parallel circulation, shutdown, and repeated examination to ensure that no significant hemorrhages occurred. For blood transfusion, the arterial cannula was later translocated to the venous cannula. The IA and the LSA artificial blood vessel were anastomosed in an end-to-end manner to complete the IA-LSA bypass operation.

An intravenous drip of protamine (in case anaphylaxis follows the operation) was also administered, followed by early removal of the venous cannula after mechanical blood transfusion, hemostasis, and chest closure. The comparison of the traditional SET and the current SET procedure is depicted in Figures 1 and 2.

\section{Statistical analysis}

Statistical analysis was conducted using SPSS 22.0 statistical 

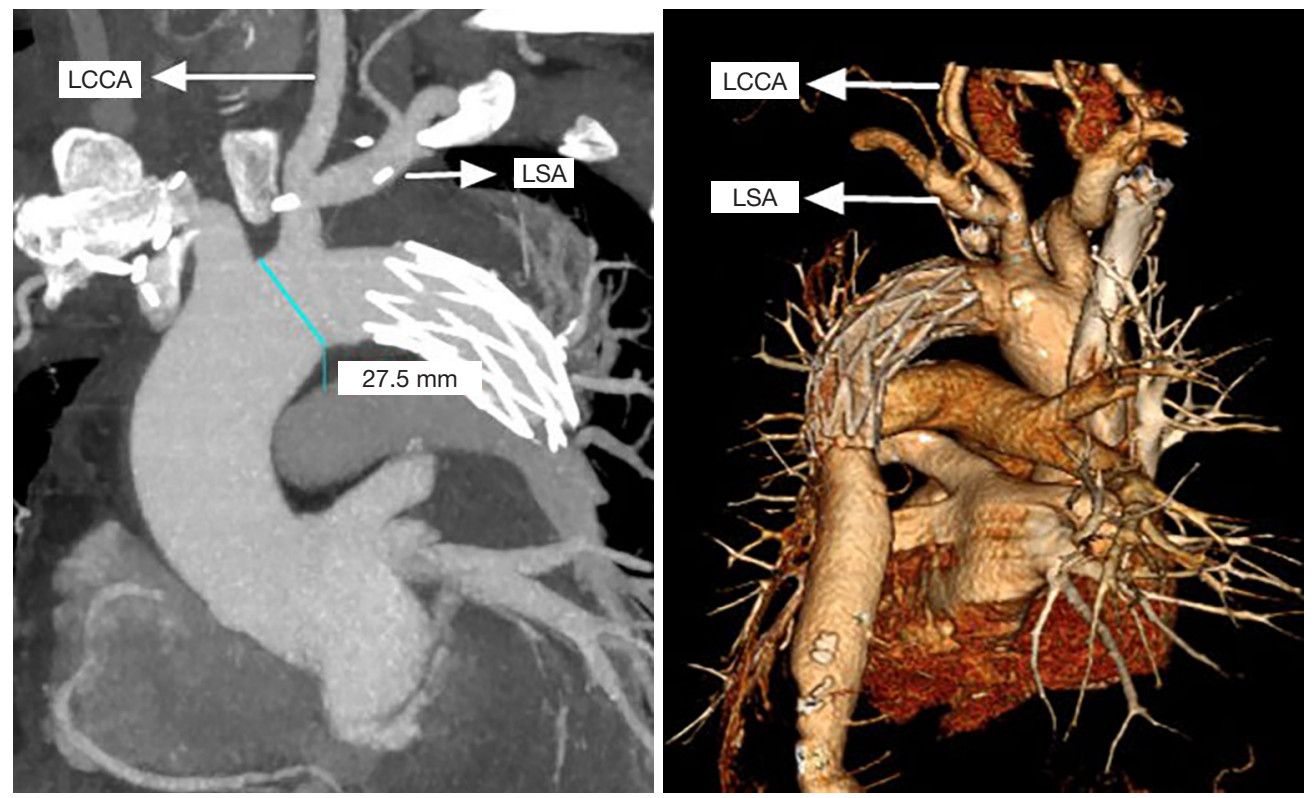

Figure 1 Traditional stented elephant trunk surgery. The left subclavian artery was anastomosed to the left common carotid artery.
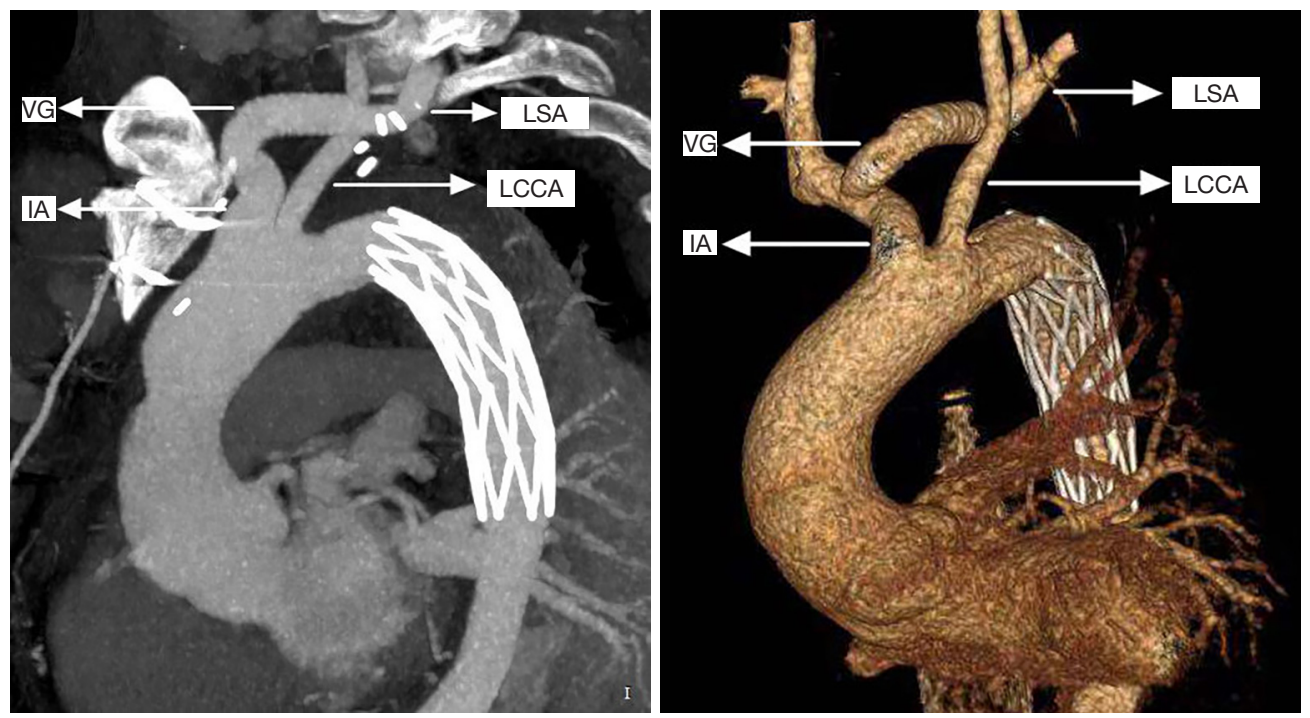

Figure 2 Modified stented elephant trunk surgery. The left subclavian artery was anastomosed to the artificial blood vessel. LSA, left collarbone artery; LCCA, left common carotid artery; IA, innominate artery; VG, artificial blood vessel.

software. The measurement data were expressed as the mean \pm standard deviation $(\mathrm{X} \pm \mathrm{s})$, and the enumeration data were expressed as the number of examples and percentages.

\section{Results}

All the patients involved in the study were successfully operated on. Operation time ranged from 4.0-5.5 $(4.65 \pm 0.47) \mathrm{h}, \mathrm{CPB}$ time was $115-163(138.6 \pm 15.64) \mathrm{min}$, aorta cross-clamping time was $32-59(42 \pm 7.72) \mathrm{min}$, and the SCP time as 15-32 (21.7 \pm 5.56$)$ min. Additionally, the cerebral perfusion flow was $5-8(6.8 \pm 1.23) \mathrm{mL} / \mathrm{kg} / \mathrm{min}$ and nasopharynx temperature was $23.5-26.8(25.22 \pm 0.96){ }^{\circ} \mathrm{C}$, while blood pressure in the left upper limb during 
deep hypothermic circulatory arrest was 20-31 (25.5 3.81) $\mathrm{mmHg}$. The intraoperative aorta cannulation was performed in a total of seven cases of IA artificial blood vessel and LSA artificial blood vessel cannulation; and three cases of IA artificial blood vessel, right femoral artery, and LSA artificial blood vessel cannulation. In addition, the post-operative ventilation time was $5-15(8.8 \pm 2.94)$ $\mathrm{h}$, retention time in ICU was 1 day; the post-operative hospitalization time was $6-8(6.9 \pm 0.74)$ days. None of the patients died, or experienced endoleakage or any related neurological complications. The closure rate of the peristent false lumen was $100 \%$, and only 1 case was reported of delayed healing after the femoral artery incision.

\section{Discussion}

Aortic dissection, a potentially catastrophic cardiovascular disease, exposes the patient to extreme lethal outcomes with an inferior natural prognosis from the onset. Sun Lizhong offered a detailed classification of aortic dissection based on the Stanford classification (5), in which type B dissection is divided into $\mathrm{B} 1, \mathrm{~B} 2$, and $\mathrm{B} 3$, according to the extent to which the descending aorta is involved. TBAD can also be sub-grouped into $\mathrm{S}$ type (simple type) and C type (complex type) based on the involvement of the aortic arch. Notably, the treatment and clinical implications of most BS-type aortic dissections are considered satisfactory after TEVAR treatment (6). However, for BC-type aortic dissection, endovascular interventional technology holds limitations for the implementation of LSA coverage, and the use of chimney technology, laser perforation technology, and Castor branched stent system due to the risk of type I endoleakage. Therefore, for younger patients who undergo BC type dissection, particularly those with Marfan syndrome, our center suggest stent elephant trunk (SET) surgery as the first choice treatment. Furthermore, patients with connective tissue diseases are likely to undergo twostage thoracoabdominal aorta replacement, where stenttype artificial blood vessels are used during the surgery to facilitate the cross-clamping and anastomosis of secondstage surgery.

SET surgery has both advantages and disadvantages in comparison with other minimally invasive surgeries involving median thoracotomy, $\mathrm{CPB}$, deep hypothermic circulatory arrest, and post-operative monitoring, which require cardiovascular surgeons to grasp a relatively longer and more difficult procedure. Therefore, a hybrid technique called axillo-axillary bypass grafting/axillary-to-carotid artery bypass grafting, TEVAR is preferred. However, the relatively high mortality and complication rates of this technique make its application in young patients with connective tissue disease who have longer life expectancies controversial (7-9). Bünger et al. (8) reported in their study involving 45 TBAD patients that hybrid surgery resulted in an in-hospital mortality rate of $11.1 \%$, early or late endoleakage rates as high as $27 \%(12 / 44)$ and $43 \%(13 / 30)$, respectively, and a re-intervention rate of $18 \%$. However, several reports have confirmed reduced operative mortality and post-operative complications with this procedure, suggesting the surgical procedure is safer and more feasible than once indicated $(10,11)$.

Nevertheless, the procedure has some long-term drawbacks. The most common among these is brachial plexus injury, in which patients suffer post-operative numbness and loss of muscle strength in their upper right limb. Most of these patients have a slower recovery rate post-treatment with neurotrophic drugs, possibly due to the traction injury of brachial plexus. A few of these patients cannot recover and their quality of life is affected. Furthermore, during the procedure, the distal axillary artery needs to be cross-clamped during axillary artery cannulation, resulting in continuous ischemia of the right upper limb, and potential limb ischemia and necrosis in extreme cases. The number of cases in this group is relatively small, with $71.4 \%(5 / 7)$ of the patients weighing more than $90 \mathrm{~kg}$, and it is now considered to be associated with aortic dissection in patients. Obesity imposes hurdles during the surgical procedure and makes it difficult to free both the axillary artery and the arterial cannulation. The resulting high pump pressure can cause injury to the axillary artery, and in extreme cases lead to dissection, which eventually results in difficulty during the surgery and risks unnecessary injury. In addition, some patients have a deep LSA which is difficult to free, while in some patients, the LSA and left common carotid artery are far apart. These two factors increase the difficulty of left common carotid artery-LSA bypass and can eventually make the patients susceptible to anastomotic stenosis or hemorrhage. Additionally, some patients have a thin left common carotid artery and thick LSA. Therefore, in our study, we explored whether the blood supply from the left common carotid artery is affected after the bypass. Importantly, during the deep hypothermic circulatory arrest, the reverse left vertebral artery steal supplies the left upper limb, although its influence on the brain's blood supply is not yet fully understood.

To solve the problems mentioned above, we implemented 
some improvements to the surgical procedure. During the operation, a section of $10 \mathrm{~mm}$ artificial blood vessel was used to anastomose the IA in an end-to-side manner via vein cannulation to avoid any possible side injury while we surgically freed the axillary artery. The anastomosis took about 5 minutes to carry out, which had little effect on the cerebral blood supply. After starting the CPB, the LSA was detached at an early stage, and the distal end was anastomosed with another $10 \mathrm{~mm}$ artificial blood vessel in an end-to-end manner and perfused by cannulation, thereby avoiding vertebral artery steal to improve cerebral blood supply. The subclavian artery was anastomosed with an artificial blood vessel, which was easy to free, and hemostasis could be performed. For the patients who simultaneously received femoral artery cannulation, post-operative treatment was relatively simple. The procedure involved bringing the temperature to $36^{\circ} \mathrm{C}$, removing of the artificial blood vessel cannula, anastomosing the artificial blood vessel in an end-to-end manner, keeping the femoral artery cannula, and performing other routine treatments. For the patients who did not receive a simultaneous femoral artery cannula, the anastomotic stoma was repeatedly examined after shutdown until there was no active hemorrhaging. Subsequently, the arterial cannula was translocated to the vena cava cannula for blood transfusion, and then the artificial blood vessel was anastomosed in an end-to-end manner. Furthermore, the neutralization of protamine was conducted slowly to avoid severe allergic reaction, and the venous cannula was removed as soon as possible after neutralization to avoid thrombosis. We performed all of these procedures on ten patients. Every patient was later discharged from the hospital without experiencing any complications of the central nervous system, or any abnormal sensation or loss of muscle strength in the right upper limb, although one patient suffered delayed healing of the femoral artery incision. There was no operative mortality or endoleakage. The closure rate of the peri-stent false lumen was $100 \%$, and there was a lower incidence of complications, suggesting that the improvements we implemented improved the safety and efficiency of the operation.

\section{Conclusions}

This study shows satisfactory results with no mortality, no endoleakage, no complications of central nervous system and no abnormal sensation or loss of muscle strength in the right upper limb which suggest this modified SET procedure is safe and effective. In practice, we found that overweight patients were more suitable for this modified procedure. However, our study has some limitations that need to be discussed. The sample size is relatively small, and there is no specific value to confirm the blood flow distribution of the three branches of the arch after the operation. Therefore, further studies involving larger sample sizes and long-term follow-up are required to verify the findings of the current study.

\section{Acknowledgments}

Funding: None.

\section{Footnote}

Conflicts of Interest: The authors have no conflicts of interest to declare.

Ethical Statement: The authors are accountable for all aspects of the work in ensuring that questions related to the accuracy or integrity of any part of the work are appropriately investigated and resolved.

Open Access Statement: This is an Open Access article distributed in accordance with the Creative Commons Attribution-NonCommercial-NoDerivs 4.0 International License (CC BY-NC-ND 4.0), which permits the noncommercial replication and distribution of the article with the strict proviso that no changes or edits are made and the original work is properly cited (including links to both the formal publication through the relevant DOI and the license). See: https://creativecommons.org/licenses/by-nc-nd/4.0/.

\section{References}

1. Daily PO, Trueblood HW, Stinson EB, et al. Management of acute aortic dissections. Ann Thorac Surg 1970;10:237-47.

2. Zhu JM, Qi RD, Liu YM, et al. Repair of complicated type $B$ dissection with an isolated left vertebral artery using the stented elephant trunk technique. Eur J Cardiothorac Surg 2016;49:782-3.

3. Chen L, Qi RD, Liu W, et al. Repair of Complicated Chronic Type B Dissection with Distal Aortic Arch Involvement Using Left Subclavian Artery Transposition with Implantation of a Stented Elephant Trunk. Thorac Cardiovasc Surg 2017;65:99-104. 
4. Zhu JM, Qi RD, Liu YM, et al. Repair of Distal Aortic Arch Aneurysms by Left Subclavian Artery Transposition with Stented Elephant Trunk Implantation. Ann Vasc Surg 2016;32:98-103.

5. Sun L, Qi R, Zhu J, et al. Total arch replacement combined with stented elephant trunk implantation: a new "standard" therapy for type A dissection involving repair of the aortic arch? Circulation 2011;123:971-8.

6. Lee M, Lee DY, Kim MD, et al. Outcomes of Endovascular Management for Complicated Chronic Type B Aortic Dissection: Effect of the Extent of Stent Graft Coverage and Anatomic Properties of Aortic Dissection. J Vasc Interv Radiol 2013;24:1451-60.

7. Schoenhoff FS, Kadner A, Czerny M, et al. Should aortic arch replacement be performed during initial surgery for

Cite this article as: Zhao H, Bai T, Xue J, Sun L, Liu Y. Application of the modified stented elephant trunk procedure in type B aortic dissection. Ann Transl Med 2020;8(6):384. doi: 10.21037/atm.2020.03.109 aortic root aneurysm in patients with Marfan syndrome? Eur J Cardiothorac Surg 2013;44:346-51.

8. Bünger CM, Carsten M, Kische S, et al. Hybrid aortic arch repair for complicated type B aortic dissection. J Vasc Surg 2013;58:1490-6.

9. Cochennec F, Tresson P, Cross J, et al. Hybrid repair of aortic arch dissections. J Vasc Surg 2013;57:1560-7.

10. Zhu J, Cheng L, Liu Y, et al. One-stage repair for stanford type B aortic dissection concomitant with cardiac diseases: open stented elephant trunk technique combined with cardiac operation. Thorac Cardiovasc Surg 2012;60:11-6.

11. Roselli EE, Bakaeen FG, Johnston DR, et al. Role of the frozen elephant trunk procedure for chronic aortic dissection. Eur J Cardiothorac Surg 2017;51:i35-9. 